\title{
Unusual clinical presentation of rare case of vaginal leiomyoma: a case report
}

\author{
Ishrat Zuber*, Purnima K. Nadkarni, Aditi A. Nadkarni, Akshay Nadkarni
}

21st Century hospital and Eva care hospital, Vapi, Gujarat, India

Received: 07 April 2016

Accepted: 07 May 2016

\section{*Correspondence:}

Dr. Ishrat Zuber,

E-mail: ishratzuber11@gmail.com

Copyright: (c) the author(s), publisher and licensee Medip Academy. This is an open-access article distributed under the terms of the Creative Commons Attribution Non-Commercial License, which permits unrestricted non-commercial use, distribution, and reproduction in any medium, provided the original work is properly cited.

\section{ABSTRACT}

Primary vaginal leiomyoma are rare and usually arise from anterior vaginal wall, approximately 330 cases of vaginal fibroid reported in world literature. Vaginal myoma usually presented as discharge per vaginum, abnormal bleeding, pain lower abdomen, dyspareunia etc. We report a case of primary vaginal leiomyoma arising from lateral vaginal wall which is presented clinically as pain in hip joint and radiating to ipsilateral leg which is unusual clinical presentation creating diagnostic dilemma and because of rarity of the case.

Keywords: Leiomyoma, Myoma, Dyspareunia, MRI, Tumor

\section{INTRODUCTION}

Vaginal tumors are rare and include papilloma, haemangioma, mucus polyp, and rarely leiomyoma. Vaginal leiomyomas remain an uncommon entity with only about 300 reported cases since the first detected case back in 1733 by Denys de Leyden, Bennett and Erlich. ${ }^{1,2}$ found only nine cases in 50,000 surgical specimens and only one case in 15,000 autopsies reviewed at Johns Hopkins Hospital. These tumors arise most commonly from the anterior vaginal wall causing varied Clinical presentation. They may or may not be associated with leiomyoma elsewhere in the body.

\section{CASE REPORT}

A 23 year female $2^{\text {nd }}$ para referred to our hospital by an orthopaedician diagnosed on Magnetic Resonance Imaging (MRI) as a case of tumor in vagina. Her last delivery was vaginal delivery in same hospital. Copper T insertion done 6 months back. She had complained of pain in her left hip joint since 6 months. pain was also radiating from Hip joint to heel .Pain was intermittent burning in character worsen in sitting for long time, initially pain relieved by local analgesics but, since last one month pain become more worse. No Complaint of
Pain Abdomen, Bleeding, Discharge or Dyspareunia. Xray hip joint was normal, MRI pelvis done which was suggestive of -Altered signal intensity lesion $5 * 5 \mathrm{~cm}$ involving left side of pelvic wall and arising from vagina with involvement of left obturator internus muscle (Figure 1). On examination small protrusion from left lateral vaginal wall seen around $3 * 4 \mathrm{~cm}$ in size. Firm to hard in consistency deeply encroaching in lateral pelvic wall. Mass was separated from cervix. Fixed to pelvic wall. On Transvaginal Sonography $4 * 5 \mathrm{~cm}$ hypoecoic mass in vagina separated from cervix and uterus seen.

Patient taken for enucleation of this fibroid with consent complete enucleation of fibroid done vaginaly. A Foley's catheter was introduced in the urethra for protecting the latter. The tumor was then sent for histopathological examination with a preoperative diagnosis of vaginal leiomyoma. Patient's intraoperative and post-operative period was uneventful. Patient discharge on $3^{\text {rd }}$ postoperative day. Gross examination revealed a $6 \times 5 \mathrm{~cm}$ solid mass with a whorling appearance in the cut section (Figure 2). Microscopic examination revealed a wellcircumscribed leiomyoma underlying the squamous epithelium, consistent with the diagnosis of vaginal leiomyoma. We follow the case for 5 month patient was comfortable in follow up. 


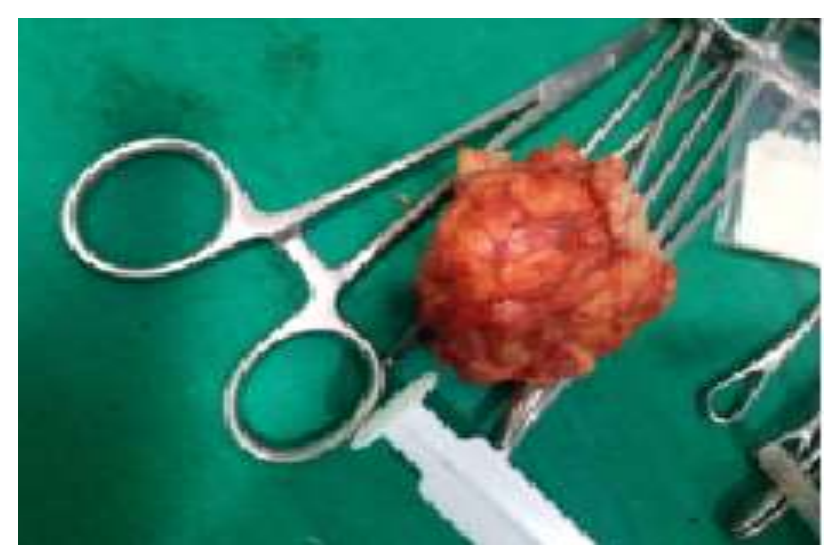

Figure 1: Gross whorled appearance of Myoma after removal.

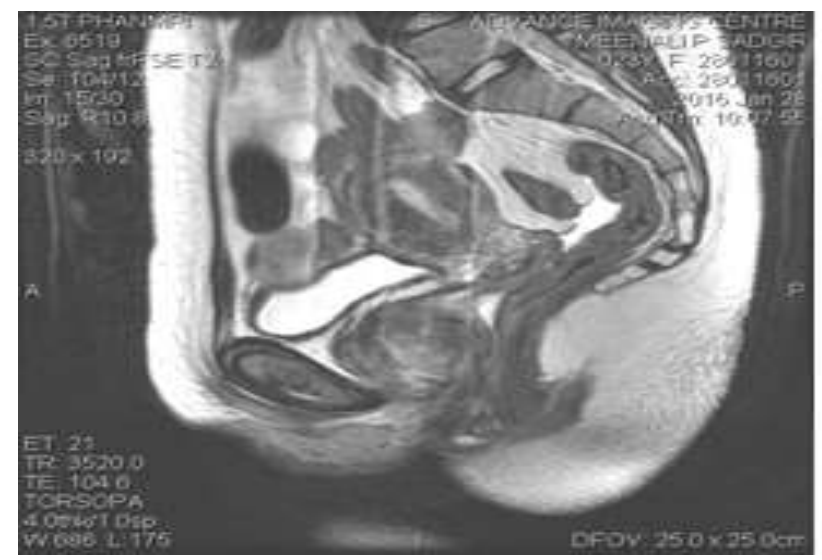

Figure 2: MRI showing vaginal mass.

\section{DISCUSSION}

Extra uterine fibroids are rare benign neoplasm which may cause diagnostic difficulties. Leiomyomas in female genital tract are common in the uterus and to some extent in the cervix followed by the round ligament, utero-sacral ligament, ovary, and inguinal canal. ${ }^{l}$ Occurrence in vagina is very rare. These are more common in women aged 30-50 years in our case is 23 year making it unusual. The clinical presentations are variable. The consistency may vary from solid to cystic and may be misleading. Patients are usually asymptomatic in early stage; Symptoms arise with the growth of tumor and mainly due to compression effect to the adjacent structure. In this case compression effect on obturator muscle and surrounding nerves presented patient as pain in hip joint like sciatica. Gouri et al reported a case in which clinical presentation was discharging gluteal swelling creating diagnostic dilemma. ${ }^{3}$ Similarly Gupta et al reported a vaginal fibroid presenting as an ovarian tumor diagnosed postoperatively. ${ }^{4}$

Imaging modalities like MRI and needle biopsy are valuable in making preoperative diagnosis. ${ }^{5}$ as in our case MRI help us to reach provisional presentation, it is difficult to differentiate between a malignant and benign tumor as well. In most cases, diagnosis is only made after histopathological examination consistent with a mixture of smooth muscle and fibrous stroma. Sarcomatous changes may occur and tumor recurrence or rapid enlargement usually indicates malignancy. ${ }^{6}$ So atypism and mitotic figure need to be evaluated in histopathology to rule out malignancy.

\section{CONCLUSION}

Surgery is recommended treatment. Malignancy should be rule out by histopathology. Surgical removal of the tumor through vaginal approach, preferably with urethral catheterization to protect the urethra during surgery, is usually the treatment of choice. In case of large tumors, however, an abdomino-perineal approach is preferred. The patient needs to be followed up for chance of recurrence. Our patient was symptom-free at 5-month follow-up.

Funding: No funding sources

Conflict of interest: None declared

Ethical approval: Not required

\section{REFERENCES}

1. Young SB, Rose PG, Reuter KL. Vaginal fibromyomata: Two cases with preoperative assessment, resection and reconstruction. Obstet Gynecol. 1991;78:972-4.

2. Bennett HG, Jr, Erlich MM. Myoma of the vagina. Am J Obstet Gynecol. 1941;42:314-20.

3. Gowri R, Soundraghavan S, Oumachigui A. Leiomyoma of the vagina: an unusual presentation. Journal of Obstetrics and Gynaecology Research. 2003;29:395-8.

4. Gupta V, Arya P. A rare case of vaginal fibroid presenting as ovarian tumour. Journal of Obstetrics Gynaecology of India. 2006;56:537-8.

5. Halder A, Mandal RD. Vaginal leiomyoma presenting as polyp. Journal of Science. 2015;10:915-6.

6. Shadbolt CL, Coakley FV, Qayyum A, Donat SM. MRI of vaginal leiomyomas. J Comput Assist Tomogr. 2001;25:355-7.

Cite this article as: Zuber I, Nadkarni PK, Nadkarni AA, Nadkarni A. Unusual clinical presentation of rare case of vaginal leiomyoma: a case report. Int $\mathbf{J}$ Reprod Contracept Obstet Gynecol 2016;5:2047-8. 